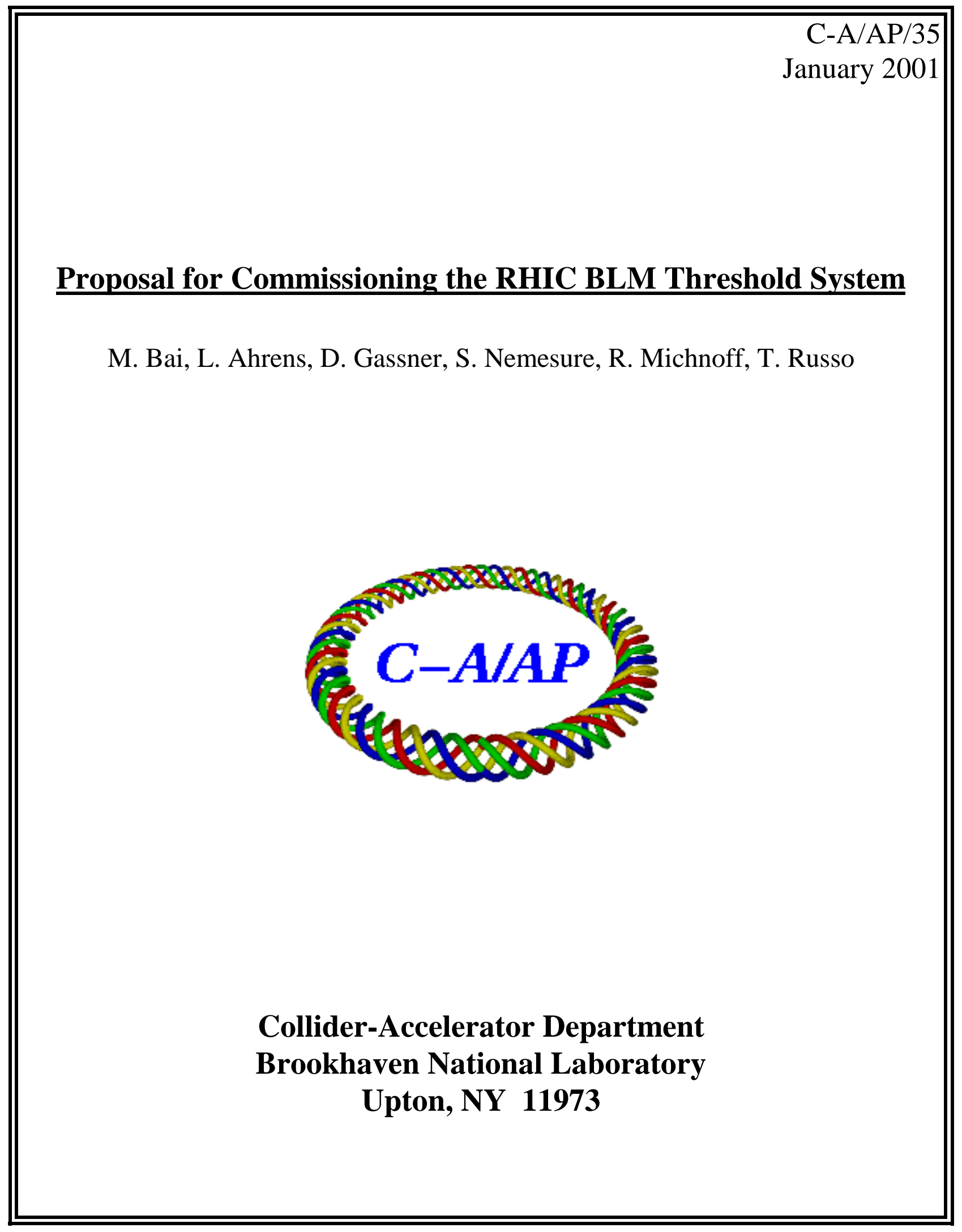




\title{
Proposal for Commissioning the RHIC BLM Threshold System
}

\author{
M. Bai, L. Ahrens, D. Gassner, S. Nemesure, R. Michnoff, T. Russo
}

January 8, 2001

\begin{abstract}
In addition to monitoring the beam losses in the ring, the RHIC loss monitor is also designed to protect the super-conducting magnets from being damaged due to an excessive beam loss. This could be either an fast beam loss or a cumulative loss over many turns. During the RHIC FY00 run, several real quench events were identified. A proposal of commissioning the RHIC loss monitor threshold system in the coming RHIC run is presented in this note.
\end{abstract}

\section{Introduction - RHIC beam loss monitor thresh- old system}

The RHIC beam loss monitor system is designed to monitor the beam loss around the ring as well as to protect the super-conducting magnets from quenching if excessive beam loss is detected. Fig. 1 is the schematic circuit diagram of an individual loss monitor. The raw signal is sent to the madc for data acquisition and the threshold system. The raw signal from the loss monitor is first conditioned by an RC filter and front end amplifier. The signal is then sent in two directions, to the madc, and to the AD734 multiplier which is the first stage of the beam inhabit threshold system. The multiplier is used to compensate the dynamic range of the signal. The gain of the AD734 multiplier is set by a 8-bit DAC. Eight multipliers on the same board share the same gain DAC.

The threshold system consists of two parts. The slow loss threshold system contains a low-pass filter with a time constant of $20 \mathrm{msec}$. It is for protecting the magnet from a cumulative beam loss over many turns. The fast loss threshold system has a high-pass filter with a time constant of $100 \mu \mathrm{sec}$ to prevent magnets from quenching due to a sudden beam crash.

The two comparators for the two sub-systems independently compare the filter output with the reference value set by a 8-bit DAC. If the detected slow or fast signal is above the reference level (threshold), a slow or fast trip bit is then 


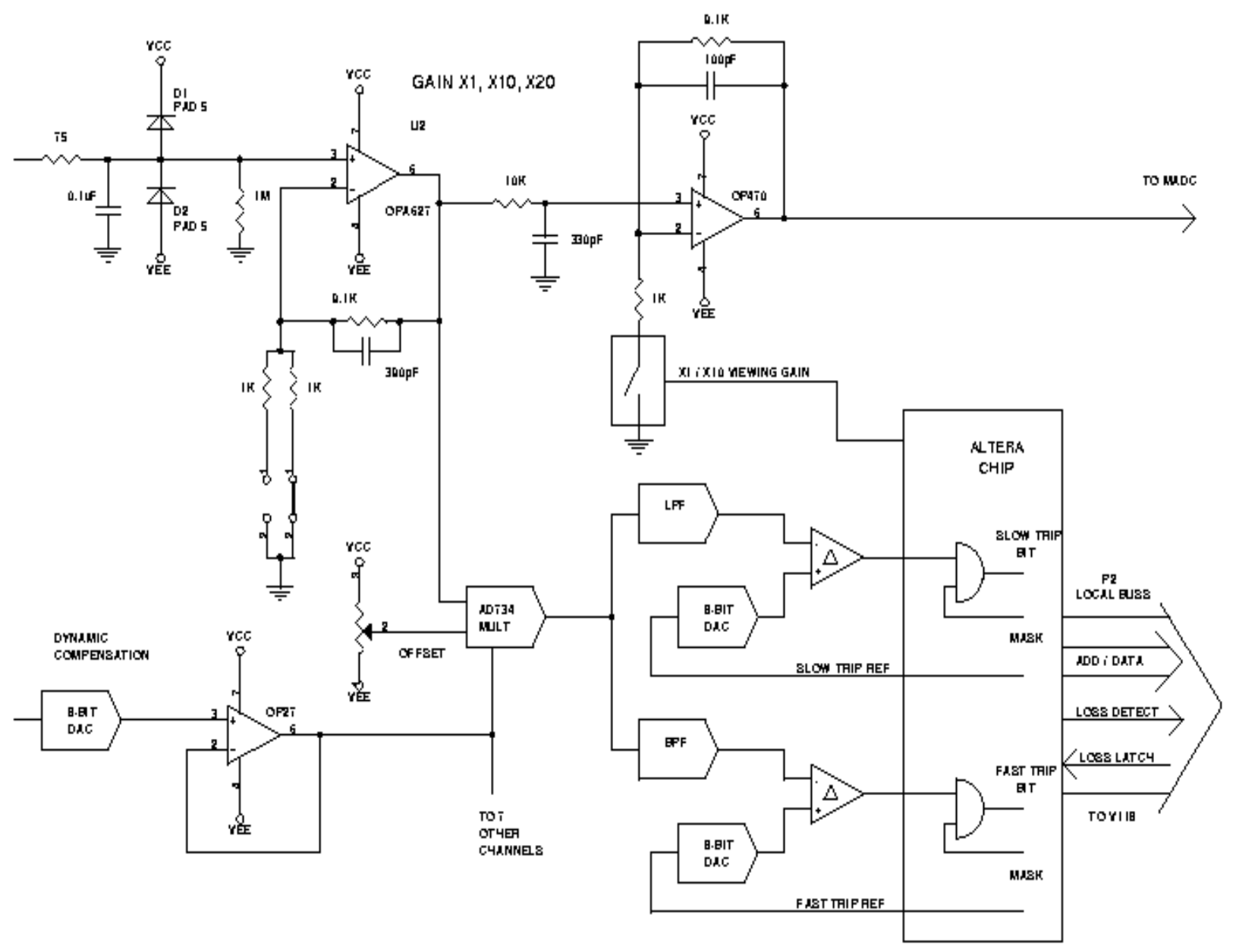

Figure 1: The schematic diagram of a typical channel of the beam loss monitor analog module.

sent out to inhibit the beam. If any trip occurs, it then latches the state of all blm's so that one can find the trip location,and do a post mortem analysis.

The slow or fast threshold level of each individual loss monitor can be individually set. The design of the system also has the freedom to mask/unmask the slow or fast comparator of each loss monitor. This is to prevent a faulty blm from inhibiting the beam or to allow special conditions. In order to accommodate the fact that the threshold level is different at different beam energy, the settings of the gains, the mask bits and the threshold values can be changed according to the RHIC events. Six events for blms are available and can be generated by the sequencer. A RHIC threshold application was developed to set up the threshold levels for all the loss monitors and also update their status [2]. 


\section{Proposal for commissioning the RHIC BLM threshold system}

The RHIC blm threshold system had gone through intensive tests without beam during the past few years. The electronics for all the channels worked very well. After careful adjustments, the threshold offsets for all the channels are positive and reasonably stable. Fig. 2 is the average of measured threshold offsets. However, due to the time constraint, the threshold system has not been commissioned with beam.

During the RHIC FY2000 run, we had several real "quench" events at beam energy $\gamma=70$ due to large beam losses [1]. Those events demonstrate the importance of the BLM threshold system. They also provide very valuable information on the threshold level and loss pattern.

Quite interesting thing is all the "real" quench events are associated with the Q2 magnets in the triplets [1]. Fig. 3 is a typical loss pattern of the "real" quench events. This data clearly indicates that the beam hit the wall of the magnet bo3-qf2 and produced large losses at the loss monitors which are down stream from the magnet. Fig. 4 is the loss monitor b3-lm0 post mortem data. This loss monitor is just down stream from the triplet. It shows that a threshold level of $2000 \mathrm{rad} / \mathrm{hr}$ at beam energy $\gamma=70$ should be sufficient to prevent the magnet from quenching.

The fact that during the run only the quadrupoles in the triplets were quenched and non-arc quadrupoles indicates that the triplet quadrupoles are effectively more fragile than the arc quadrupoles. Those "real" quench events also demonstrate the relevance of the threshold system. Especially, for the coming run, higher intensity will be expected. To have a reliable working threshold system will be a good insurance. Hence, we would like to propose to first commission the slow threshold system of the blms around the IR regions with beam. Our plan is;

1. Enable the slow and fast threshold system for the loss monitors around the IR regions and Disable the blm threshold inputs from the beam permit system. Set the slow trip level to $2000 \mathrm{rad} / \mathrm{hr}$ and for the fast trip level to $200 \mathrm{rad}$ [5] for all the energies. Monitor the threshold status for a week to see whether there is any beam inhibit due to the misfiring of the loss monitor threhsold system.

2. Enable the blm threshold in the permit system and keep the threshold settings at $2000 \mathrm{rad} / \mathrm{hr}$.

3. Use two blm event links to set different trip levels at $\gamma=70$ and top energy if the blm post mortem data shows the magnets quench at a level lower than $2000 \mathrm{rad} / \mathrm{hr}$ for $\gamma>70$. In general, one expects the relation of the 
threshold and the beam energy to be $2000 *\left(\frac{67.8}{E_{k}}\right)^{0.8} \mathrm{rad} / \mathrm{hr}[3]$ where $E_{k}$ is the kinetic energy in $\mathrm{GeV}$.

4. If time allows, we would like to use a corrector to create a beam loss in the middle of an arc and acquire beam loss data for the arc quadrupoles. This will require about a 8 -hr beam study time.

5. For the fast threshold system, we will first study the fast loss signal at injection using the loss monitors around the injection area. Some dedicated end-of-store time will also be very valuable for studying the response of the fast threshold system at the top energy. For this, we would like to use the abort kicker to create fast losses around the triplet area. Ref. [1] shows that a too weak kick puts the beam into the triplet on 11 o'clcok side of IP12.

6. Dedicated beam time at higher energy is also desired to commission the fast threshold system if time permits.

(a) At top energy, use abort kicker to create a fast loss at the triplet area and use oscilloscope to record the fast beam losses.

(b) Repeat this for different beam intensity until a real quench is created, record the beam intensity and the level of fast beam losses.

(c) Repeat the study around Romen Pots area using a local corrector instead of the abort kicker. However, it is a slow loss due to the limit of the corrector ramp rate. The alternative is to move the crystal at the scraper area into the beam to create a large quick beam loss around that area [4].

\section{Conclusion}

The RHIC loss monitor threshold system has not been used for the past two runs of RHIC. Several real quench events at beam energy of $\gamma=70$ happened during the latest RHIC run. This fact, plus the higher stored energy and full intensity in the coming run emphasizes the necessity of having a reliable working RHIC loss monitor threshold system.

\section{References}

[1] L. Ahrens, Five Quenches during the 2000 RHIC Gold Run, C-A/AP/31, 2000.

$[2]$ http://www.rhichome.bnl.gov/Controls/doc/RhicLossThreshold/RhicLossThreshold.html.

[3] discussion with A. Stevens. 
[4] discussion with A. Drees.

[5] Witkover, R. L., et al., "RHIC Beam Loss Monitor System Initial Operation", Proc 1999 PAC p2247. 

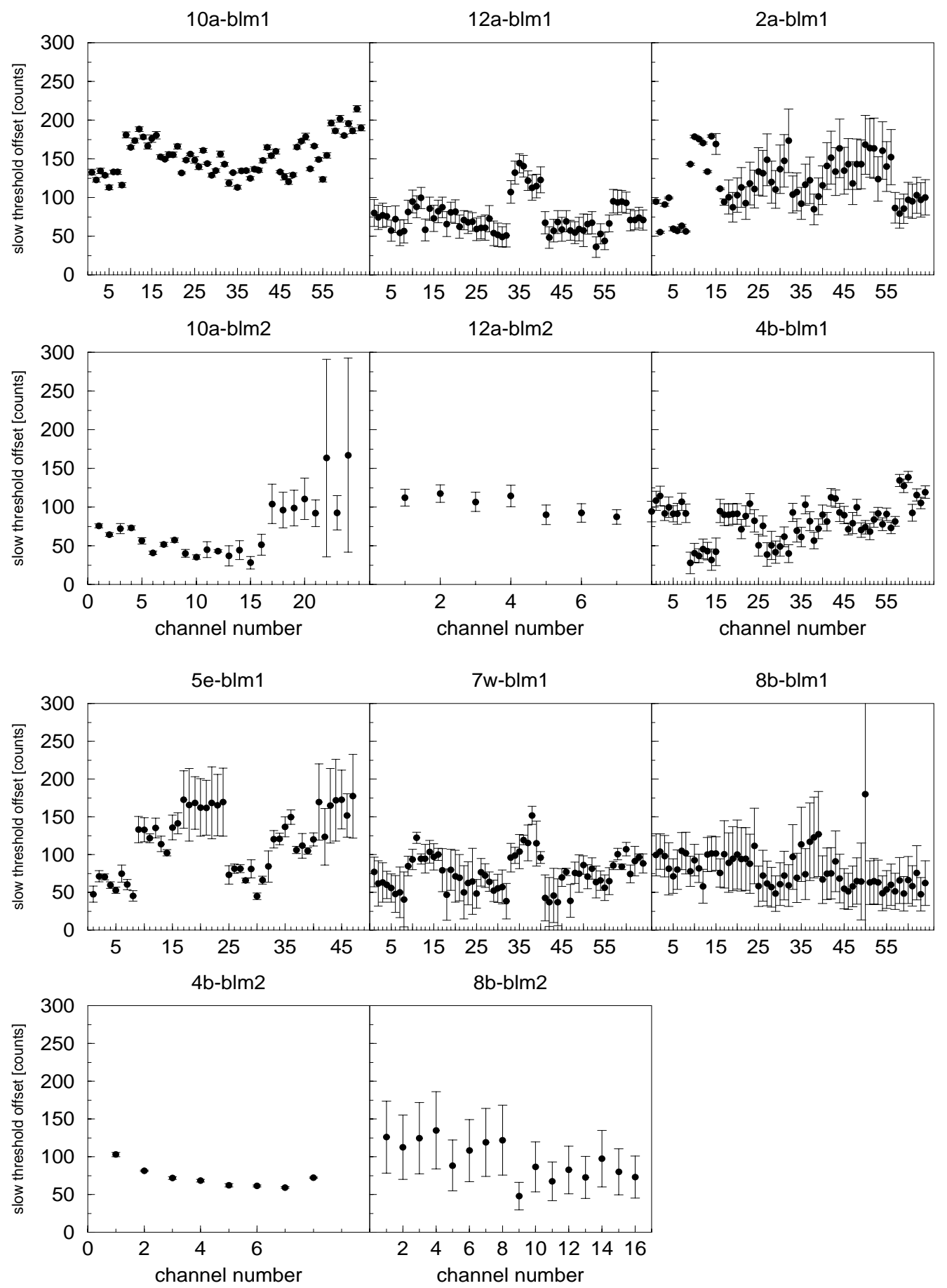

Figure 2: Beam loss monitor slow threshold offsets averaged over eight difference measurements. The measurements were taken over a 3 week time span. The gains were all set at 1 count. 

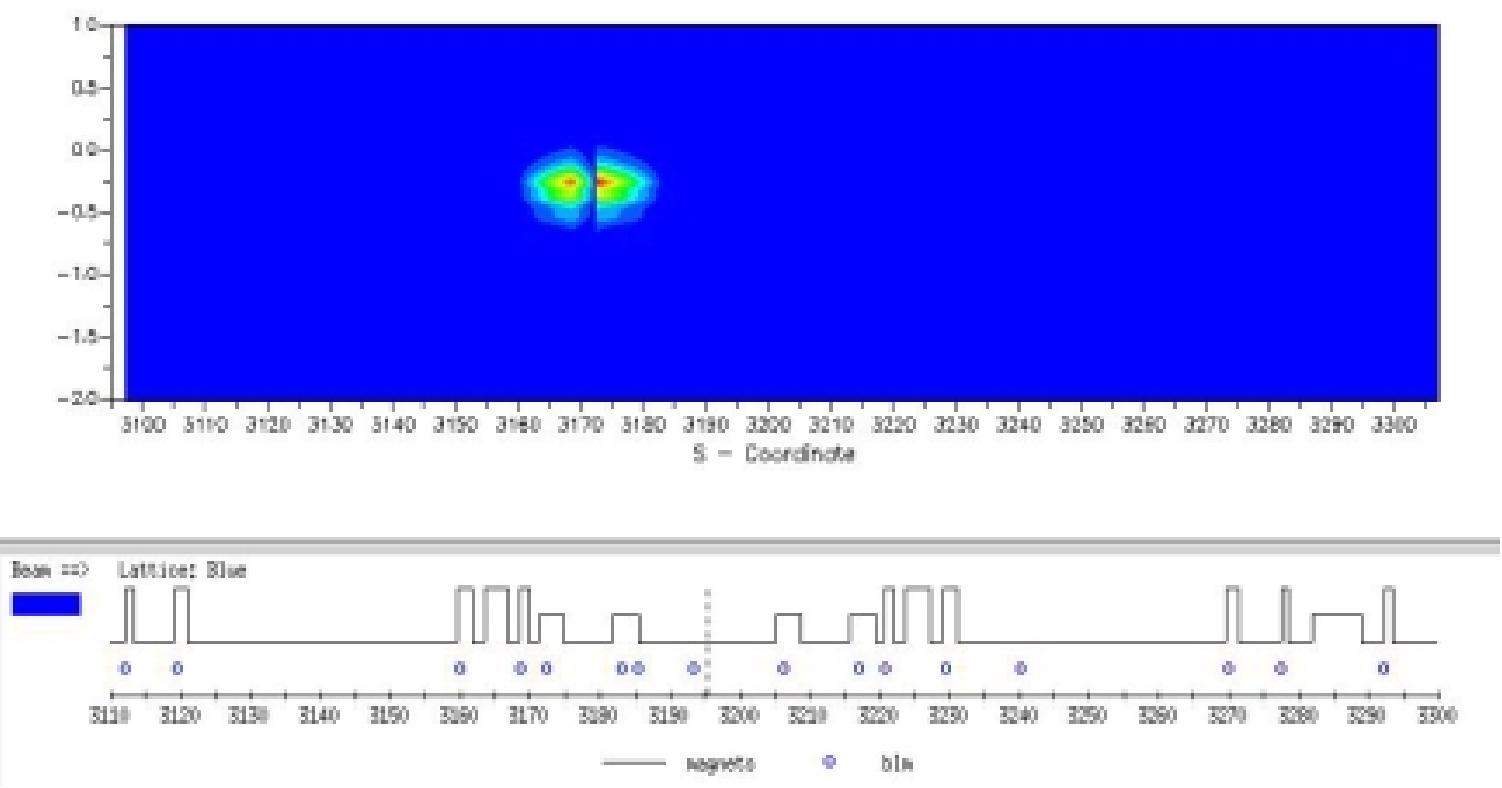

Figure 3: b3-lm0 post mortem data

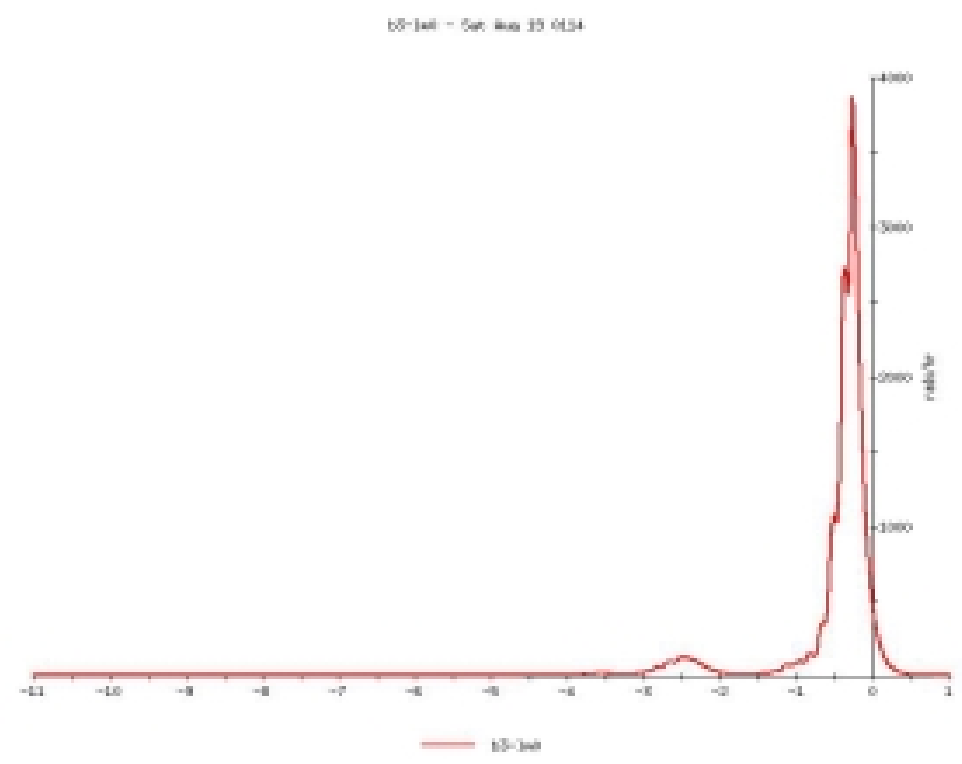

Figure 4: Beam loss pattern around IP4 during the quench event at 01:14 on Aug. 19, 2000. The horizontal axis is the time in seconds. Time 0 second corresponds to the time when the permit was pulled by the magnet quench link. 